\title{
EKSISTENSI BAGIAN IMAJINER PADA INTEGRAL FORMULA INVERSI FUNGSI KARAKTERISTIK
}

\author{
YULIANA PERMATASARI \\ Program Studi Matematika, \\ Fakultas Matematika dan Ilmu Pengetahuan Alam, Universitas Andalas Padang, \\ Kampus UNAND Limau Manis Padang, Indonesia \\ anazharian@gmail.com
}

Abstrak. Formula inversi dari fungsi karakteristik $\varphi_{X}(t)$ dengan fungsi distribusi $F$ adalah

$$
F(x)-F(0)=\lim _{T \rightarrow \infty} \frac{1}{2 \pi} \int_{-T}^{T} \frac{\exp (-i t x)-1}{-i t} \varphi_{X}(t) d t
$$

untuk setiap $-\infty<x<\infty$.

Syarat perlu dan cukup untuk

$$
\lim _{T \rightarrow \infty} \operatorname{Im}\left(\frac{1}{2 \pi} \int_{0}^{T} \frac{\exp (-i t x)-1}{-i t} \varphi_{X}(t) d t\right)
$$

ada adalah

ada, dimana

$$
\lim _{\varepsilon \rightarrow 0^{+}} \int_{\varepsilon}^{\infty} \frac{G(u, x)-(u, 0)}{u} d u
$$

$$
G(u, x)=F(u+x)-F(-u+x)
$$

Kata Kunci: Fungsi distribusi, fungsi karakteristik, formula inversi.

\section{Pendahuluan}

Misalkan $F(x)$ adalah fungsi distribusi dan $\varphi_{X}(t)$ fungsi karakteristik yang didefinisikan sebagai berikut

$$
\varphi_{X}(t)=E\left[e^{i t X}\right]
$$

di mana $e^{i t X}=\cos (t X)+i \sin (t X)$. Formula inversi dari fungsi karakteristik $\varphi_{X}(t)$, yaitu

$$
F(x)-F(0)=\lim _{T \rightarrow \infty} \frac{1}{2 \pi} \int_{-T}^{T} \frac{e^{-i t x}-1}{-i t} \varphi_{X}(t) d t .
$$

Formula inversi (1) dapat dipisahkan menjadi bagian real dan bagian imajiner, yaitu

$$
\operatorname{Re}\left(\frac{1}{2 \pi} \int_{0}^{T} \frac{\exp (-i t x)-1}{-i t} \varphi_{X}(t) d t\right)=\operatorname{Re}\left(\frac{1}{2 \pi} \int_{-T}^{0} \frac{\exp (-i t x)-1}{-i t} \varphi_{X}(t) d t\right)
$$

dan

$$
\operatorname{Im}\left(\frac{1}{2 \pi} \int_{0}^{T} \frac{\exp (-i t x)-1}{-i t} \varphi_{X}(t) d t\right)=-\operatorname{Im}\left(\frac{1}{2 \pi} \int_{-T}^{0} \frac{\exp (-i t x)-1}{-i t} \varphi_{X}(t) d t\right)
$$


karena bagian imajiner pada $\frac{1}{2 \pi} \int_{-T}^{T} \frac{e^{-i t x}-1}{-i t} \varphi_{X}(t) d t$ tidak selalu ada, dalam paper ini dibahas kajian eksistensi integral pada formula inversi untuk bagian imajiner.

\section{Eksistensi Bagian Imajiner Pada Integral Formula Inversi Fungsi Karakteristik}

Teorema 2.1. [3] Syarat perlu dan cukup untuk

$$
\lim _{T \rightarrow \infty} \operatorname{Im}\left(\frac{1}{2 \pi} \int_{0}^{T} \frac{\exp (-i t x)-1}{-i t} \varphi_{X}(t) d t\right)
$$

ada adalah

$$
\lim _{\varepsilon \rightarrow 0^{+}} \int_{\varepsilon}^{\infty} \frac{G(u, x)-(u, 0)}{u} d u
$$

ada, di mana

$$
G(u, x)=F(u+x)-F(-u+x)
$$

Bukti. Perhatikan bahwa bentuk integral (3) ada pada lingkungan tak hingga. Kemudian misalkan

$$
G(u, x)-G(u, 0)=[F(u+x)-F(u)]-[F(-u+x)-F(-u)]
$$

dan

$$
F(x+u)-F(u) \in L(-\infty, \infty)
$$

Misalkan

$$
I(x, T)=\operatorname{Im}\left(\frac{1}{2 \pi} \int_{0}^{T} \frac{\exp (-i t x)-1}{-i t x} \varphi_{X}(t) d t\right) .
$$

Dapat dilihat bahwa

$$
\begin{aligned}
I(x, T) & =\frac{1}{2 \pi} \int_{-\infty}^{\infty} d F(u) \int_{0}^{T} \frac{\sin x t \sin u t-\cos u t+\cos x t \cos u t}{t} d t \\
& =\frac{1}{2 \pi} \int_{-\infty}^{\infty} d F(u) \int_{0}^{T} \frac{\cos (u-x) t-\cos u t}{t} d t \\
& =\frac{1}{2 \pi} \int_{-\infty}^{\infty} d F(u) \int_{0}^{T} d t \int_{u-x}^{u} \sin t v d v \\
& =\frac{1}{2 \pi} \int_{-\infty}^{\infty} d F(u) \int_{u-x}^{u} \frac{1-\cos v T}{v} d v \\
& =\frac{1}{2 \pi} \int_{-\infty}^{\infty} \frac{1-\cos v T}{v} d v \int_{v}^{v+x} d F(u) \\
& =\frac{1}{2 \pi} \int_{0}^{\infty}[G(v, x)-G(v, 0)] \frac{1-\cos v T}{v} d v .
\end{aligned}
$$

Berdasarkan Lema Riemann-Lebesgue [2] maka

$$
\lim _{T \rightarrow \infty} \int_{\varepsilon}^{\infty}[G(v, x)-G(v, 0)] \frac{\cos v T}{v} d v=0 \text { untuk setiap } \varepsilon>0 .
$$


Untuk $\varepsilon>0$ sebarang dan $T \rightarrow 0$ dapat ditulis bahwa

$$
I(x, T)=\int_{0}^{\varepsilon} \frac{[G(v, x)-G(v, 0)]}{v}(\cos v T) d v+\int_{\varepsilon}^{\infty} \frac{[G(v, x)-G(v, 0)]}{v} d v+o(1)
$$

$(\Rightarrow)$ Misalkan terdapat sebarang $\varepsilon>0$ dan

$$
\begin{aligned}
& \int_{0}^{\varepsilon} \frac{G(v, x)-G(v, 0)}{v}(1-\cos v T) d v \\
& =\int_{0}^{1 / T}+\int_{1 / T}^{\varepsilon}=K_{1}+K_{2},
\end{aligned}
$$

di mana

$$
\begin{aligned}
K_{1} & =\int_{0}^{1 / T} G(v, x)-G(v, 0) \frac{1-\cos v T}{v} d v \\
& \leq C T \int_{0}^{1 / T}|G(v, x)-G(v, 0)| d v
\end{aligned}
$$

untuk suatu konstanta $C$. Karena $F$ fungsi tak turun maka $\lim _{v \rightarrow 0}[G(v, x)-$ $G(v, 0)]=0$, sehingga (8) konvergen ke nol. Jadi dapat diperoleh

$$
K_{1}=o(1) \quad \text { untuk } \quad T \rightarrow \infty .
$$

Definisikan

$$
\chi(v)=G(v, x)-G(v, 0) .
$$

Pilih $\varepsilon>0$ sedemikian sehingga $|\chi(v)|<\delta$ untuk $|v| \leq \varepsilon$, untuk sebarang $\delta$. Karena $\chi(v) / v$ terbatas pada $[1 / T, \varepsilon]$, dengan menggunakan Teorema Nilai Tengah Kedua [1] diperoleh

$$
\begin{aligned}
K_{2} & =\int_{1 / T}^{\varepsilon} G(v, x)-G(v, 0) \frac{1-\cos v T}{v} d v \\
& \leq \int_{1 / T}^{\varepsilon} \frac{\chi(v)}{v}-T \chi\left(\frac{1}{T}\right) \int_{1 / T}^{\xi} \cos v T d v-\left(\frac{\chi(\varepsilon)}{\varepsilon}\right) \int_{\xi}^{\varepsilon} \cos v T d v
\end{aligned}
$$

untuk $1 / T<\xi<\varepsilon$. Maka

$$
\left|K_{2}-\int_{1 / T}^{\varepsilon} \frac{\chi(v)}{v} d v\right| \leq 2 \chi\left(\frac{1}{T}\right)+2 \chi(\varepsilon) \leq 4 \delta
$$

Oleh karena (6) dan (7) diperoleh

$$
\left|I(x, T)-\frac{1}{2 \pi} \int_{1 / T}^{\infty} \frac{\chi(v)}{v} d v\right| \leq \frac{2 \delta}{\pi}+o(1) .
$$

Ini menunjukkan syarat cukup dan sekaligus menyatakan (3) ada.

$(\Leftarrow)$ Misalkan $\chi(v)$ didefinisikan seperti (11). Dapat dilihat bahwa $\lim _{v \rightarrow 0^{+}} \chi(v)=c$. Jika $c \neq 0$, maka dengan menggunakan (6) diperoleh

$$
I(x, T)-c \int_{0}^{\varepsilon} \frac{1-\cos v T}{v} d v=\int_{0}^{\varepsilon} \frac{[\chi(v)-c](1-\cos v T)}{v} d v+\int_{0}^{\infty} \frac{\chi(v)}{v} d v+o(1) .
$$


42 Yuliana Permatasari

Perhatikan bahwa

$$
\begin{aligned}
& \int_{0}^{\varepsilon} \frac{[\chi(v)-c](1-\cos v T)}{v} d v \\
& =\int_{0}^{1 / T}+\int_{1 / T}^{\varepsilon}=L_{1}+L_{2}
\end{aligned}
$$

diperoleh

$$
\begin{aligned}
L_{1} & =\int_{0}^{1 / T} \frac{[\chi(v)-c](1-\cos v T)}{v} d v \\
& \leq C T \int_{0}^{1 / T}|\chi(v)-c| d v
\end{aligned}
$$

Dari (14) diperoleh

$\left|I(x, T)-\frac{c}{2 \pi} \int_{0}^{\varepsilon} \frac{1-\cos v T}{v} d v-\frac{1}{2 \pi} \int_{1 / T}^{\varepsilon} \frac{\chi(v)}{v} d v-\frac{1}{2 \pi} \int_{\varepsilon}^{\infty} \frac{\chi(v)}{v} d v\right| \leq C_{1} \delta+o(1)$,

untuk suatu konstanta $C_{1}$.

$$
\int_{0}^{\varepsilon} \frac{1-\cos v T}{v} d v=2 \int_{0}^{\varepsilon T} \frac{\sin ^{2} v / 2}{v} d v \geq C_{2} \log \varepsilon T,
$$

untuk suatu konstanta $C_{2}$. Pilih sebarang $\varepsilon>0$, diberikan $\eta<C_{2}$ sedemikian sehingga $|\chi(v)-c|<\eta$ untuk $0<v<\varepsilon$. Maka

$$
\left|\int_{1 / T}^{\varepsilon} \frac{\chi(v)-c}{v} d v\right| \leq \eta \log \varepsilon T .
$$

Jika limit $I(x, T)$ ada untuk $T \rightarrow \infty$, maka karena (16) dan (17), implikasi (15) kontradiksi. Oleh karena itu maka $c=0$, sehingga

$$
\left|I(x, T)-\frac{1}{2 \pi} \int_{1 / T}^{\infty} \frac{\chi(v)}{v} d v\right| \leq C_{1} \delta+o(1) .
$$

Syarat perlu terbukti.

\section{Ilustrasi}

Fungsi karakteristik dari sebaran $U(0,1)$ adalah $\varphi_{X}(t)=i(1-\exp (i t)) / t$ dengan fungsi distribusi $F(x)=x$. Maka formula inversi dari sebaran $U(0,1)$ adalah

$$
\begin{aligned}
F(x)-F(0)= & \lim _{T \rightarrow \infty} \frac{1}{2 \pi} \int_{-T}^{T} \frac{\exp (-i t x)-1}{-i t} \varphi_{X}(t) d t \\
= & \lim _{T \rightarrow \infty} \frac{1}{2 \pi} \int_{-T}^{T} \frac{\cos (t-t x)-\cos t x-\cos t+1}{t^{2}} d t \\
& +i \lim _{T \rightarrow \infty} \frac{1}{2 \pi} \int_{-T}^{T} \frac{\sin t x+\sin (t-t x)+\sin t}{t^{2}} d t
\end{aligned}
$$


Diperoleh

$$
I(x, T)=\lim _{T \rightarrow \infty} \frac{1}{2 \pi} \int_{0}^{T} \frac{\sin t x+\sin (t-t x)+\sin t}{t^{2}} d t
$$

Kemudian akan ditunjukkan

$$
\lim _{T \rightarrow \infty} \operatorname{Im}\left(\frac{1}{2 \pi} \int_{0}^{T} \frac{\exp (-i t x)-1}{-i t} \varphi_{X}(t) d t\right)=\int_{0}^{\infty} \frac{G(u, x)-G(u, 0)}{u} d u
$$

Perhatikan bahwa,

$$
\begin{aligned}
I(x, T) & =\lim _{T \rightarrow \infty} \frac{1}{2 \pi} \int_{0}^{T} \frac{\sin t x+\sin (t-t x)+\sin t}{t^{2}} d t \\
& =\lim _{T \rightarrow \infty} \frac{1}{2 \pi} \frac{-\cos T x+\cos (-T+T x)+x T \sin T+1-\cos T}{t^{3}} d t \\
& =0
\end{aligned}
$$

dan

$$
\begin{aligned}
G(u, x) & =F(u+x)-F(-u+x) \\
& =2 u \\
G(u, 0) & =F(u+0)-F(-u+0) \\
& =2 u
\end{aligned}
$$

Sedemikian sehingga diperoleh,

$$
\begin{aligned}
\lim _{\varepsilon \rightarrow 0^{+}} \int_{\varepsilon}^{\infty} \frac{G(u, x)-G(u, 0)}{u} d u & =\lim _{\varepsilon \rightarrow 0^{+}} \int_{\varepsilon}^{\infty} \frac{2 u-2 u}{u} d u \\
& =0
\end{aligned}
$$

Dalam hal ini dapat dilihat bahwa,

$$
\lim _{T \rightarrow \infty} \operatorname{Im}\left(\frac{1}{2 \pi} \int_{0}^{T} \frac{\exp (-i t x)-1}{-i t} \varphi_{X}(t) d t\right)=\lim _{\varepsilon \rightarrow 0^{+}} \int_{0}^{\infty} \frac{G(u, x)-G(u, 0)}{u} d u
$$

\section{Ucapan Terima kasih}

Penulis mengucapkan terima kasih kepada Bapak Dodi Devianto, Bapak Admi Nazra, Ibu Hazmzra Yozza, Ibu Lyra Yulianti, dan Bapak Syafrizal Sy, yang telah memberikan masukan dan saran sehingga makalah ini dapat diselesaikan dengan baik.

\section{Daftar Pustaka}

[1] Bartle, R.G. dan Donald R.S. 1927. Introduction to Real Analysis, $2^{\text {nd }}$ Edition. John Wiley and Sons Inc., Singapore.

[2] Jain, P.K. dan V.P. Gupta. 1976. Lebesgue Measure and Integration. Wiley Eastern Limited, New Delhi.

[3] Kawata, T. 1969. On The Inversion Formula For The Characteristic Function. Pacific Journal of Mathematics. 